\title{
Flow, force, behaviour: assessment of a prototype hydraulic barrier for invasive fish
}

\author{
Joschka Wiegleb $(\mathbb{D} \cdot$ Philipp E. Hirsch $(\mathbb{D}$ - Frank Seidel • Georg Rauter $(\mathbb{D} \cdot$ \\ Patricia Burkhardt-Holm (i)
}

Received: 13 July 2021 / Revised: 27 October 2021/Accepted: 10 November 2021/Published online: 3 December 2021

(C) The Author(s) 2021

\begin{abstract}
Migration barriers being selective for invasive species could protect pristine upstream areas. We designed and tested a prototype protective barrier in a vertical slot fish pass. Based on the individuals' swimming responses to the barrier flow field, we assumed this barrier would block the ascension of the invasive round goby, but allow comparable native species (gudgeon and bullhead) to ascend. The barrier was tested in three steps: flow description, quantification of forces experienced by preserved fish in the flow field, and tracking the swimming trajectories of ca. 43 live fish per trial and species. The flow and the forces
\end{abstract}

Handling editor: Grethe Robertsen

Supplementary Information The online version contains supplementary material available at https://doi.org/10.1007/ s10750-021-04762-z.

J. Wiegleb · P. E. Hirsch · P. Burkhardt-Holm ( $\square)$ Department of Environmental Sciences, Program ManSociety-Environment, University of Basel, Vesalgasse 1, 4051 Basel, Switzerland

e-mail: patricia.holm@unibas.ch

F. Seidel

Institute for Water and River Basin Management, Karlsruhe Institute of Technology, Kaiserstrasse 12, 76131 Karlsruhe, Germany

G. Rauter

BIROMED-Lab, Department of Biomedical Engineering, University of Basel, Gewerbestrasse 14, 4123 Allschwil, Switzerland were homogenous over the barrier, though gudgeon experienced significantly smaller forces than round goby or bullhead. The swimming trajectories were distinct enough to predict the fish species with a random forest machine learning approach (92.16\% accuracy for gudgeon and $85.24 \%$ for round goby). The trajectories revealed round goby and gudgeon exhibited increased, but varied, swimming speeds and straighter paths at higher water discharge. These results suggest that passage of round goby was prevented at $130 \mathrm{~L} / \mathrm{s}$ water discharge, whereas gudgeon and bullhead could pass the barrier. Our findings open a new avenue of research on hydraulic constructions for species conservation.

Keywords Tracking $\cdot$ Hydraulic forces $\cdot$ Swimming performance $\cdot$ Fish pass $\cdot$ Invasive species $\cdot$ Random forest

\section{Introduction}

The majority of the world's rivers are fragmented by anthropogenic barriers (Belletti et al., 2020). The passage of fish across such barriers is crucial to ecological river connectivity and the functionality of aquatic ecosystems (Silva et al., 2018). Traditionally, various types of fish passes have been designed to support the passage of economically relevant fish 
species (Katopodis and Williams, 2012). However, fish pass design increasingly needs to respond to the demands for protection of specific species (United Nations, 1992). This often means meeting the individual requirements of native species for conservation purposes and, at the same time, hindering the upstream migration of invasive species. Therefore, a combination of research approaches from fluid dynamics, engineering and behavioural ecology is necessary to account for the individual differences in swimming performance between species (Kemp, 2012). This idea has been implemented in studies applying robotics to describe basic fish swimming kinematics (Thandiackal et al., 2021) or studies linking flow measurements with the swimming behaviour of fish (Drucker and Lauder, 1999; Sagnes and Statzner, 2009; Porreca et al., 2017). Subsequent studies have focused on fish pass hydraulics (Larinier, 2008; Tsikata et al., 2014; Baki et al., 2017) or species compositions and fish swimming behaviour in fish passes (Jansen et al., 1999; Aarestrup et al., 2003; Knaepkens et al., 2005).

Substantial advances in our understanding of hydrodynamics in fish passes have been achieved in recent years (Wang et al., 2010; Puertas et al., 2004; Bombač et al., 2014; Fuentes-Pérez et al., 2018), including estimates of passage rates based on computational modelling and flow velocity measurements (Plesiński et al., 2018; Sanchez et al., 2020). While some studies have numerically derived the hydrodynamics of fish from flow observations (Drucker and Lauder, 1999; Lauder and Madden, 2007; Carlson and Lauder, 2011), direct empirical measures of the forces experienced by fish in flowing water remain scarce (though see Van Wassenbergh et al., 2015; QuicazanRubio et al., 2019 for recent advances).

To fill this knowledge gap on direct quantifications of the physical impact of flow on the swimming behaviour of fish, we combined hydrodynamics and fish behaviour observations into a unified three-step approach (Fig. 1) by characterising the flow field created by a specific fish pass design (Step 1), determining the physical effects of the flow on preserved individuals of specific target species (Step 2 ), and describing the behavioural responses of live fish in the same flow field (Step 3). We proposed this combinatory flow force behaviour approach would facilitate characterization of the functionality of fish
Fig. 1 Schematic illustration of the experimental steps performed in this study to assess a hydraulic barrier for round goby (grey arrows). We included a hydrodynamic assessment (Step 2) in the common methodological approach (dashed grey arrow): adaptation of the flow within the fish pass design following flow description (Step 1) and design evaluation based on the behaviour of live fish (Step 3). The components assessed in each step of this study are highlighted in red and the direction of flow is represented by the black arrow

pass designs and enable more precise evaluations of the suitability of prototype barriers for specific target species.

We applied this approach in the context of the upstream migration of the invasive round goby [Neogobius melanostomus (Pallas, 1814)]. The round goby is a very successful aquatic invasive species that has populated numerous North American and European aquatic habitats (Kornis et al., 2012; AdrianKalchhauser et al., 2020). The upstream range expansion of this species into ecologically valuable headwaters is a severe environmental threat, that can lead to important and irreversible ecosystem impacts (Phillips et al., 2003; Myles-Gonzalez et al., 2015; Ramler and Keckeis, 2020; Šlapanský et al., 2020). Ensuring the passage of native species and impeding the passage of invasive species over river obstacles is a major challenge for decision makers and requires advances in integrated interdisciplinary research (Rahel and McLaughlin, 2018). Hoover et al. (2003) tested the upstream swimming capabilities of round goby and questioned whether a hydraulic barrier could stop the upstream range expansion of this species. The same authors reported a hydraulic barrier for round goby - a bottom-dwelling species-would require an increased flow velocity, as well as an extended length of smooth substrate (Hoover et al., 2003). The design of a hydraulic barrier, similar to Kerr et al. (2021), that can prevent the upstream movement of round goby, would be simple to realize, but a ground-breaking achievement in conservation science. This idea of a selective hydraulic barrier has been implemented in our prototype.

The present study follows our previous fish ecological experiments, in which we compared the upstream swimming performance of the round goby and two native species that inhabit the same or similar 


\section{Step 1: Flow modulation and description}

-We changed the fish pass design to modulate the flow in a vertical slot fish pass

- The flow was modulated by a prototype hydraulic barrier for the invasive round goby

- An acoustic Doppler was applied to measure the flow velocity in speed $[\mathrm{m} / \mathrm{s}]$ and the Turbulent Kinetic Energy $[\mathrm{J} / \mathrm{Kg}]$

\section{Step 2: Experienced forces depending on shape}

- Forces are assumed a direct measure of the species-specific hydrodynamic burden the fish experience over the barrier

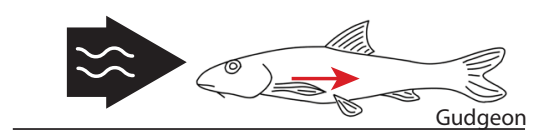

- Individual shape characteristics were assumed to determine the experienced forces

-The forces $[\mathrm{N}]$ experienced by preserved fish in the modulated flow were measured for every species

\section{Step 3: Species-specific behaviour}

- The behavioural response of live fish to the modulated flow field was assessed with video records and tracking

- The tracking paths of the live fish were used to compare various indices describing tracking courses

-We compared numerical data of the species-specific swimming behaviour with the hydraulics and hydrodynamics measured in Step 1 and 2 to describe the effect of flow on the swimming behaviour 
habitats and are described as benthic (bullhead, Cottus gobio Linnaeus, 1758) or semi-pelagic [gudgeon, Gobio gobio (Linnaeus, 1758)] swimmers (Egger et al., 2020). Based on these initial observations of swimming performance and upstream dispersal success, we now aimed to achieve a deeper mechanistic insight into the parameters that determine the speciesspecificity of a hydraulic barrier by comparing the impact of a prototype barrier vertical slot fish pass model on round goby, gudgeon, and bullhead.

This evaluation fills an important research gap by providing data on a key component required to understand the behavioural responses of fish swimming in flow: the force the flow exerts on the fish body. For example, Li et al. (2021) investigated the swimming behaviour of Schizothorax prenanti (Tchang, 1930) when swimming upstream a vertical slot and found behavioural adaptation to local flow patterns. We assumed that this behavioural response to flow might depend on individual body shape characteristics of the species. Therefore, we measured the forces experienced by real, preserved fish bodies to compare the hydrodynamic burden the different species experience based on the sum of their morphological characteristics (Wiegleb et al., 2020). Such variations in the forces experienced and swimming behaviour between species could provide the basis of a barrier prototype that provides species-specific passage.

The scientific logic behind our approach was to assess the functionality of the barrier with application of the three steps mentioned above: We described the flow to check whether we created a homogenous flow field over the barrier. The force measurements were expected to show differences in the hydrodynamics induced by the flow field between the species. We then compared the forces experienced by the fish with the swimming behaviour of live fish over the barrier to check whether the forces experienced by the fish over the barrier had an effect on the swimming behaviour. If yes, we assumed the barrier design created hydraulic conditions affecting the swimming behaviour of the tested fish species differently, impairing the passage of round goby whilst allowing native species to ascend in the best case. Overall, the research question was: Can the hydrodynamics within a barrier prevent the upstream passage of round goby whilst allowing the passage of native, comparable species?
Fig. 2 (A) Vertical slot fish pass model with the prototype selective barrier $(\mathrm{Ba})$ positioned between partition walls (W1, $W 2$ ). The fish were released at the downstream end of the model near the grid $(\mathrm{G})$ and free to swim in the upstream direction (M) against the flow (F). Two cameras ( $1, C 2$, view is represented by the black contours) recorded fish behaviour at the barrier. One camera frame (from camera $\mathrm{C} 1$ ) is provided in D showing one round goby passing the barrier (white ellipse). The left and right edges of the screen are curved as the footage was undistorted to enable tracking. The forces acting on preserved fish (a round goby connected to the sensor labelled $\mathrm{P}$ in $\mathbf{B}$ ) were measured using a probe $(\mathbf{C})$. The probe consisted of a force sensor (Se), which was connected to the $10 \mathrm{~cm}$ long fixation stick $(\mathrm{St})$ via a mounting plate $(\mathrm{Mp} 1)(\mathbf{E})$. The sensor was mounted on a mounting plate (Mp2) which was connected on a stable aluminium rod ( $\mathrm{R})$. We used a polyvinyl-chloride tape (Ta) to protect the sensor cable against damage from the aluminium rod holding the sensor. This rod was surrounded by a polyvinyl-chloride hull $(\mathrm{H})$, which shielded the sensor from surrounding flow. The hull could be opened for maintenance by a screw connection (Sf) and the sensor cable (Ca) left the probe at the top of the probe. For the measurements, the preserved fish were connected with screws to the fixation stick

\section{Materials and methods}

Fish catch, maintenance, and ethical approval

We analysed video recordings of the swimming behaviour of live fish from a previous study (Egger et al., 2020) (experiments at Karlsruhe Institute of Technology). Bullhead and gudgeon were sampled in the River Alb in Karlsruhe by means of electrofishing for this study. The fish were immediately transported to the Karlsruher Institute of Technology, Germany (KIT). Round goby were sampled between 22 and 29 March 2019 in the High Rhine in Basel, Switzerland, using minnow traps baited with dog food (Frolic $\AA$ ) and transported to the KIT. Details about the fish catchments and maintenance are provided in Egger et al. (2020).

\section{Barrier design and flow channel setup}

All experiments were performed using a vertical slot fish pass model (scale 1:1.6) at the Theodor-Rehbock Hydraulic Engineering Laboratory at the KIT (Fig. 2). To record fish movements, we installed cameras in one vertical slot of the fish pass, above the prototype hydraulic barrier. The hydraulic barrier consisted of a three-dimensional stainless-steel structure (length: $1 \mathrm{~m}$, width: $24 \mathrm{~cm}$, roughness $[k]=0.015 \mathrm{~mm}$ ) made 


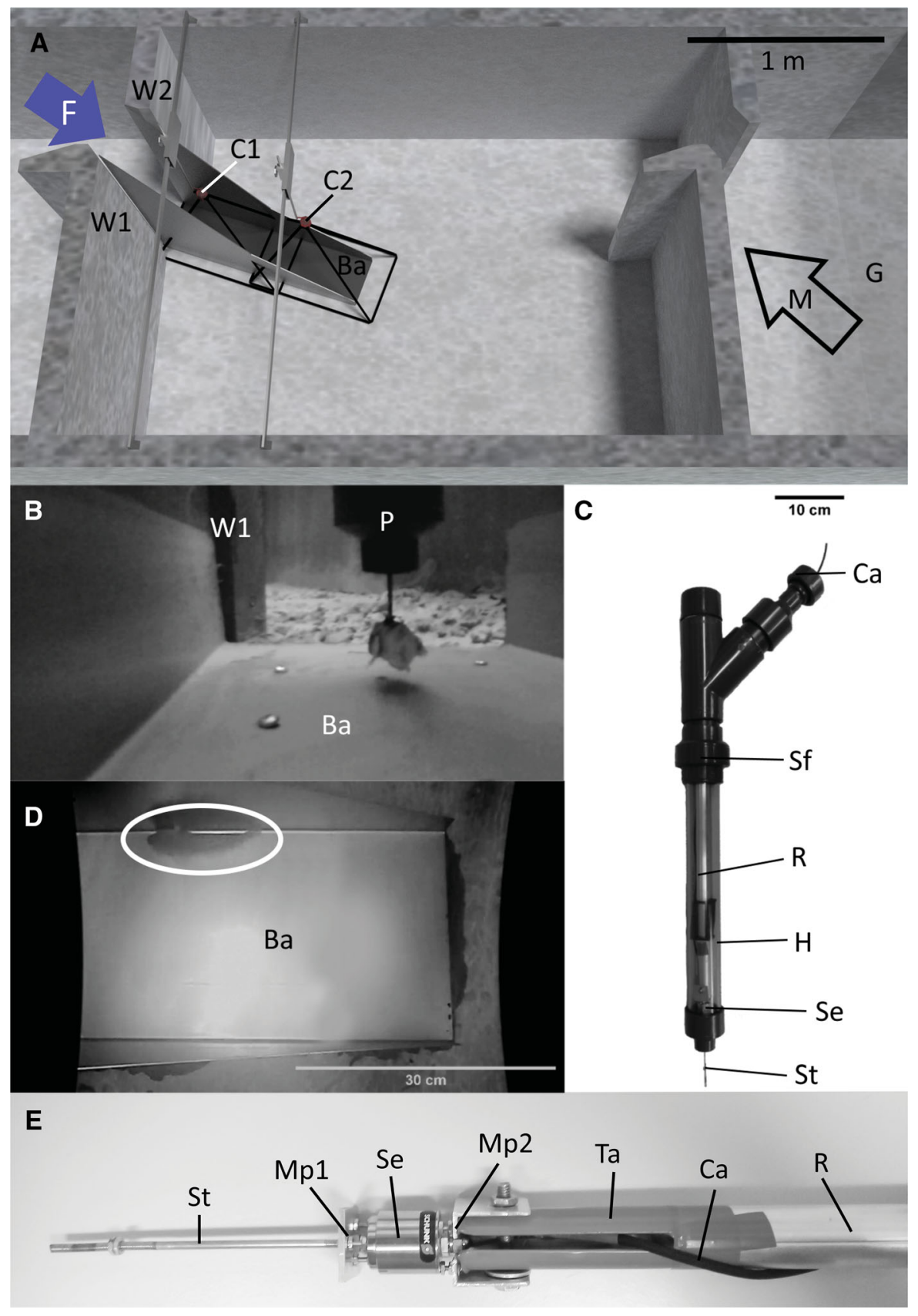

of $3 \mathrm{~mm}$ sheets. The hydraulic barrier was aligned to the direction of flow in the vertical slot fish pass and placed at an angle of 70 degrees to the partition walls to extend the field with the highest flow velocity created by the partition walls. To force the fish to travel the full length of the smooth barrier surface and maintain the withholding effect of the barrier, the barrier had two sidewalls that prevented fish from entering the barrier from the side. The height of the lateral walls of the barrier decreased with the direction of flow $[0.75 \mathrm{~m}$ at the slot, $0.20 \mathrm{~m}$ in the centre (located $0.55 \mathrm{~m}$ from the end of the structure)] to increase stability of the barrier in the flow and to avoid flapping of the side walls (Fig. 2). The barrier was 
designed to geometrically separate the outflowing jet from the water body in the basin and the corresponding shear layer and prevent the fish from station holding due to its smooth surface. The experiments followed the protocol described by (Egger et al., 2020). After adjustment to the desired water discharge rate, the fish were released at the downstream end of the fish pass and able to move within the setup for two hours unaffected by human presence. Video footage was recorded for subsequent analysis. Because water discharges can vary in real vertical slot fish passes, we included three different water discharges $(80,105$ and $130 \mathrm{~L} / \mathrm{s}$ ) in our experiments. The two lower discharge rates were chosen to increase the probability of recording migration behaviour of the live fish, because, based on previous research, they did not pose a challenge to the swimming capacities of the tested species (Egger et al., 2020). The $130 \mathrm{~L} / \mathrm{s}$ water discharge was included in the experiment because we found this discharge led to the most representative flow velocities compared to actual best-practice vertical slot fish passes (Bombač et al., 2017); thus, we focused our analyses on the data collected at $130 \mathrm{~L} / \mathrm{s}$ water discharge. Flow and behavioural data were collected for all water discharges tested, whilst force data were only collected at 130 and $80 \mathrm{~L} / \mathrm{s}$.

Step 1: measurement of flow in the prototype hydraulic barrier

The velocity in the prototype barrier was measured at 14 points (Fig. 3) as close as possible to the ground $(2-3 \mathrm{~cm})$ using an acoustic Doppler ADV probe (Vectrino, Nortek) for five minutes at every point at a nominal velocity range of 1.0 and $2.5 \mathrm{~m} / \mathrm{s}$, measurement volume of $7 \mathrm{~mm}^{3}$ and sampling rate of $25 \mathrm{~Hz}$. Flow data were processed using WinADV32
(V.2.031) and MATLAB 2019 to compute mean velocities, standard deviations and turbulent kinetic energy (TKE) for comparison with the force data (Supplementary Material 1).

\section{Step 2: measurement of forces experienced}

by preserved fish in the prototype barrier

Fish from previous behaviour experiments were euthanized with an overdose of MS 222 after the live fish swimming experiment, transferred to the lab at the University of Basel on ice and preserved in formalin and ethanol as described previously (Wiegleb et al., 2020) (non-spread fins treatment). These preserved fish were employed in our experiments, rather than 3D-printed models of scanned fish in other studies (Van Wassenbergh et al., 2015), to provide a closer approximation of real fish bodies.

For the force measurements, we selected similarly sized samples of the three fish species. To account for variation within species, we replicated the force measurements using 5-7 individuals $(n=7$ at 130 $\mathrm{L} / \mathrm{s}$ and $n=5$ at $80 \mathrm{~L} / \mathrm{s}$ water discharge) of similar size-classes (see below) for every species. The fixation stick for the force measurements was inserted in the assumed centre of gravity of the fish body (QuicazanRubio et al., 2019). The mean wet weight $\left(W_{\mathrm{w}}\right)$ of round goby was $18.14 \mathrm{~g}$ ( \pm 9.01 standard deviation [SD]) and the mean total length $\left(T_{\mathrm{L}}\right)$ was $11.0 \mathrm{~cm}$ ( $\pm 1.7 \mathrm{SD})$. The mean $W_{\mathrm{w}}$ of gudgeon was $9.78 \mathrm{~g}$ $( \pm 2.89 \mathrm{SD})$ and the mean $T_{\mathrm{L}}$ was $11.6( \pm 1.0 \mathrm{SD})$, whilst the mean $W_{\mathrm{w}}$ of bullhead was $10.31 \mathrm{~g}( \pm 3.19$ $\mathrm{SD})$ and the mean $T_{\mathrm{L}}$ was $9.8 \mathrm{~cm}( \pm 1.0 \mathrm{SD})$.

The forces acting on the preserved round goby, bullhead, and gudgeon in the flow field were measured using a water-resistant (IP 68) Nano17 Multi-Axis-F/ T-Sensor (Schunk $\left.{ }^{\circledR}\right)$ to determine the forces in three

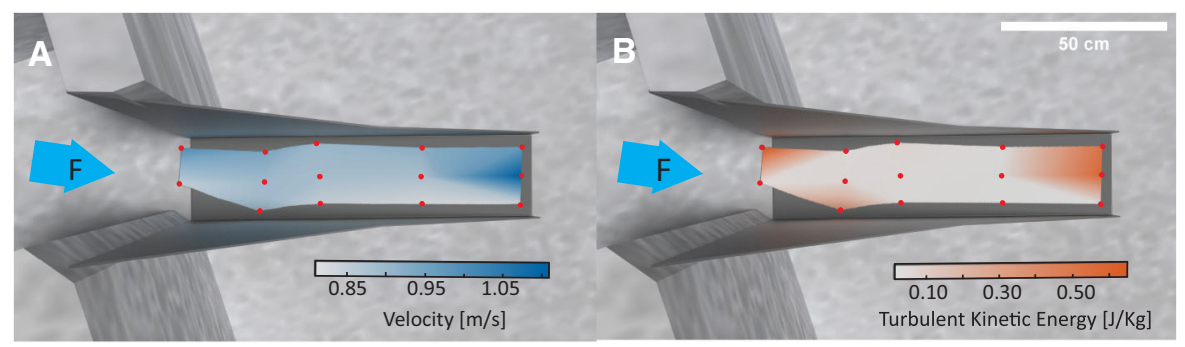

Fig. 3 Flow velocity (A) and turbulent kinetic energy (B) over the prototype barrier at a water discharge of $130 \mathrm{~L} / \mathrm{s}$. Measurement points are indicated by the red spots and the flow direction is shown by the blue arrow labelled F. See Supplementary Material 3 for the flow data at 105 and $80 \mathrm{~L} / \mathrm{s}$ water discharge 
dimensions at a sampling rate of $1000 \mathrm{~Hz}$. The multiaxis-force-torque-sensor was integrated in a stable PVC (polyvinyl-chloride) probe (Fig. 2), which sheltered the sensor against flow. The probe was installed at the same electronic carriage used for the flow measurements and approached the same measurement positions to ensure maximal comparability between force- and flow measurements. When submerging the sensor over the first measurement point, a ventile at the top of the probe was opened manually, allowing the water to enter the probe. When the water level inside the probe reached the outside water level, we closed the ventile for the duration of the experimental run until the probe was lifted out of the water. This avoided water level, and thereby pressure, fluctuation in the probe which would have had an impact on the force measurement.

There was a connection from the sensor to the tested fish via a $10 \mathrm{~cm}$ long and $3 \mathrm{~mm}$ thick brass fixation stick (Fig. 2). This stick acted as a lever, which transduced the force acting at the fish to the sensor, similar to earlier research (Wiegleb et al., 2020). For the measurement, the fish was positioned over the measurement point with a distance of 1 to $2 \mathrm{~cm}$ to the ground. This distance was chosen to avoid physical contact between fish and bottom, because this would affect the force measurement through friction forces. The fish were always oriented with the head against the flow in the same angle as the hydraulic barrier $\left(70^{\circ}\right.$ to the partition walls).

The detected forces in three directions $\left(F_{X}\right.$ : cranialcaudal axis of the fish, $F_{Y}$ : left lateral and right lateral side of the fish, $F_{Z}$ : vertical axis) were used to compute the force acting at one time in three-dimensional space applying the Pythagorean Theorem, resulting in the 3D-force $\left(F_{3 \mathrm{D}}\right)$.

$F_{X Y}=\sqrt[2]{F_{X}^{2}+F_{Y}^{2}}$

$F_{3 D}=\sqrt[2]{F_{X Y}^{2}+F_{Z}^{2}}$

With $F_{X Y}$ being the force experienced by the fish on the $F_{X}$ and $F_{Y}$ plane (Supplementary Material 2, 3).

After transformation of the measured forces (force $[\mathrm{N}]^{-1.55}$ ) to approximate normal distributions, repeated measures one-way ANOVA was performed for the forces measured at different positions and pairwise comparisons were used to test for differences between species. Spearman's rank correlations were applied to test the relationships between the forces acting on the fish and flow measured at the corresponding measurement position.

\section{Step 3: observation of the behaviour of live fish over the prototype barrier}

To obtain a deeper understanding of the dependency of swimming behaviour on the flow conditions and the forces experienced by the preserved fish bodies in flow, we recorded the swimming behaviour of 39-45 live fish per species (round goby $T_{\mathrm{L}}$ $=10.43 \mathrm{~cm} \pm 1.28 \quad \mathrm{SD}, \quad$ gudgeon $T_{\mathrm{L}}$ $=11.46 \mathrm{~cm} \pm 1.13 \quad \mathrm{SD}$ and bullhead $T_{\mathrm{L}}=9.91 \mathrm{~cm} \pm 1.22 \mathrm{SD}$ ) on the hydraulic barrier prototype. The behaviour of the fish was recorded using two IP 68 cameras (Security-Center IR CCTVCamera, 380 TV-lines; Abus, Wetter, Germany) positioned vertically over the barrier (40 and $42 \mathrm{~cm}$ above). The cameras were placed in the maximum vertical distance away from the barrier to achieve a large field of view whilst keeping the lenses of the cameras under water to obtain clear footage with minimal air bubble impact. The videos were recorded using Debut v 5.46 (C) NCH software and video processing was performed in Blender 2.79 (Community, 2017). With this program, the fish trajectories were manually recorded and event types were classified by one investigator as 'passage', 'uncompleted passage', 'return', 'uncompleted return' and 'approach' according to the criteria described in Supplementary Material 4.

In a previous study, we showed that the numbers of each type of event differed significantly between species at a water discharge rate of $130 \mathrm{~L} / \mathrm{s}$; no passages of round goby or bullhead through the barrier prototype were observed, whilst gudgeon were able to pass the barrier (Egger et al., 2020), whilst we focused on the inter-species variation in the swimming trajectories in the present study. Therefore, we recorded the trajectories of the fish on and near the barrier (Supplementary Materials 5) and extracted the following features reported by McLean and Volponi (2018) from every trajectory to characterize the swimming behaviour: mean swimming speed $[\mathrm{m} / \mathrm{s}]$, variation in speed represented by the $\mathrm{SD}$ of the swimming speed $[\mathrm{m} / \mathrm{s}]$, maximum acceleration $\left[\mathrm{m} / \mathrm{s}^{2}\right]$, distance covered [cm], sinuosity and straightness (McLean and Skowron Volponi, 2018) 

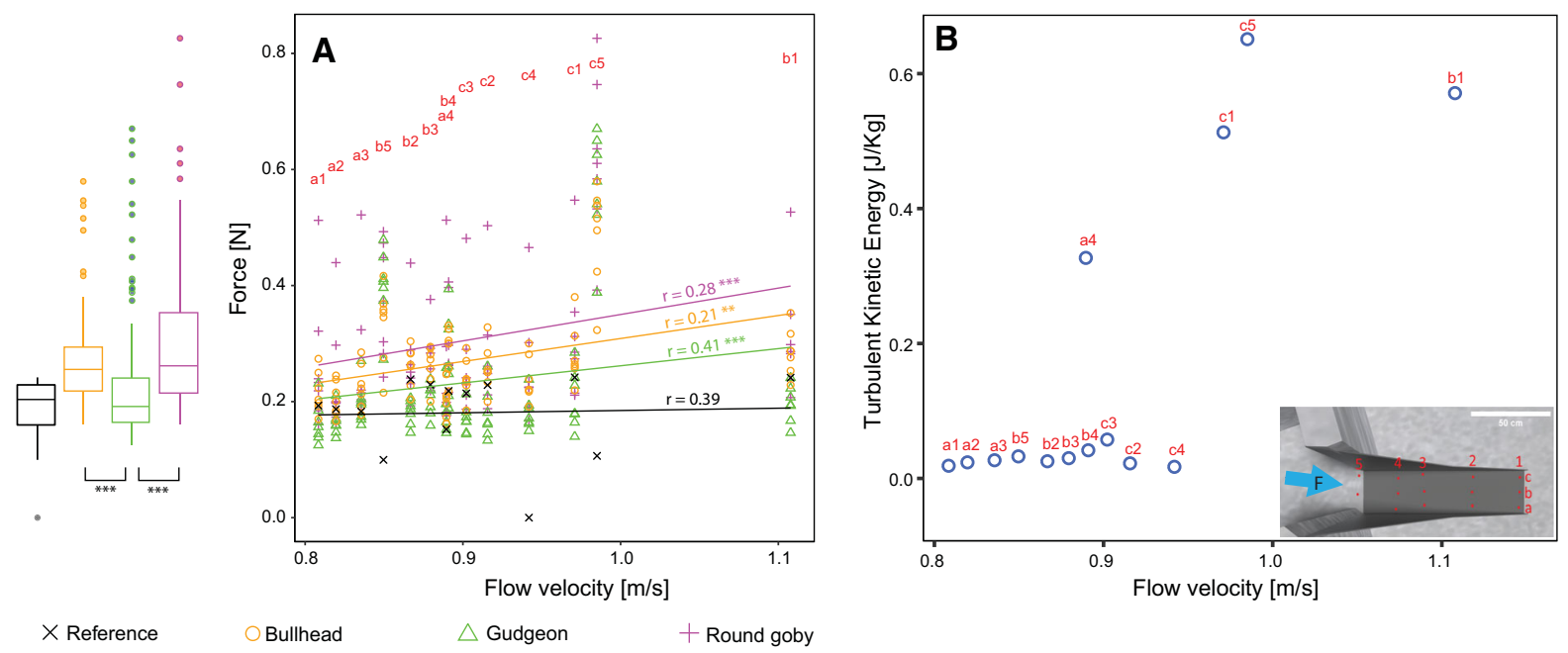

Fig. 4 Correlation between flow velocity and the forces experienced by preserved fish (seven fish per species) (A) and correlation between flow velocity and turbulent kinetic energy $(r=0.78, p<0.01)($ B) at a water discharge of $130 \mathrm{~L} / \mathrm{s}$. The boxplots on the left side of $\mathbf{A}$ represent the force distributions detected for the three fish species and the reference (one

(Supplementary Material 6, 7, 8). Previous research showed that these features provide in-depth insight into the movements of animals (McLean and Volponi, 2018). Subsequently, we performed principal component analysis, similarly to McLean and Volponi (2018), to reduce the number of dimensions and identify the features that explain most of the variation in the data; $95 \%$ confidence ellipses were computed to visually compare the behaviour events observed for each species.

We then used a random forest model to mathematically assess whether the swimming trajectories are distinct enough between species to allow for a discrimination between species. The model ('randomForest' package in R) was designed, created, trained and validated to predict the fish species (response variable) exclusively with information about the trajectory features, event type and water discharge (predictor variables):

$$
\begin{aligned}
& \text { species } \sim \text { flow }+ \text { event type }+ \text { mean swimming speed } \\
& + \text { variation in swimming speed }+ \text { maximum acceleration } \\
& + \text { distance covered }+ \text { sinuosity }+ \text { straightness }
\end{aligned}
$$

The model consisted of 50,000 trees with 6 (out of 8) variables randomly sampled at every node. To assess the model including all trajectories available, we experimental run without fish). Statistically significant differences between the boxplots and regression lines are marked by asterisks $(* p<0.1$; ** $p<0.05 ; * * * p<0.01)$. The location and designation of the measurement points are provided in the lower right inset of $\mathbf{B}$, with the arrow labelled $\mathrm{F}$ representing the direction of flow

performed cross validation and split the dataset (131 trajectories) in three subsets of similar size (two subsets with 44 and one subset with 43 trajectories) with randomly selected tracks and equal proportion of trajectories recorded for round goby $(47.33 \%)$, gudgeon $(38.93 \%)$ and bullhead $(13.74 \%)$ in every subset. This variation in trajectory frequency resulted from the varying number in the total data set of trajectories recorded for the different species. In the following, we combined two subsets to one training set, which was used for training the model. This model was then validated by the third subset, which represented the test set. In sum, we trained the model three times with every subset serving as test set once it was trained with different combinations of the other subsets (Fig. 6).

Because we knew the species for all trajectories due to our study design, we were able to determine the mean accuracy over all trained models as the frequency of correctly predicted species from the total number of predictions. The importance of trajectory features were determined by the mean decrease in accuracy when excluding the variable whilst training [mda]. The 'approach' tracks reduced the model performance and were therefore excluded from the random forest approach. We included exclusively trajectories recorded over the barrier in this analysis. 


\section{Results}

Step 1: flow over the prototype barrier

The flow velocity varied over the barrier in terms of both speed (mean $0.91 \mathrm{~m} / \mathrm{s} \pm 0.08 \mathrm{SD}$ ) and TKE (mean $0.17 \mathrm{~J} / \mathrm{Kg} \pm 0.24 \mathrm{SD}$ ) (Supplementary Material 9). The flow speed was lower over the centre of the barrier than at both ends of the barrier, where we observed strong increases in velocity $(1.11 \mathrm{~m} / \mathrm{s}$ at position b1 and $0.99 \mathrm{~m} / \mathrm{s}$ at position $\mathrm{c} 5$ ) and increases in TKE $(0.66 \mathrm{~J} / \mathrm{Kg}$ at $\mathrm{c} 5,0.58 \mathrm{~J} / \mathrm{Kg}$ at $\mathrm{b} 1,0.51 \mathrm{~J} / \mathrm{Kg}$ at c1 and 0.33 at a4; Fig. 3, Fig. 4B). The flow measurements revealed a moderate correlation between flow velocity and TKE $(r=0.78, p<0.01$; Fig. 4B) at $130 \mathrm{~L} / \mathrm{s}$ water discharge (which is assumed representative for a prototype fish pass), indicating that the small-scale hydraulic properties (described by TKE) are not well represented in the mean velocities measured at the chosen points.

At $105 \mathrm{~L} / \mathrm{s}$ water discharge, we observed a mean velocity of $0.70 \mathrm{~m} / \mathrm{s} \pm 0.05 \mathrm{SD}$ with a mean TKE of $0.02 \mathrm{~J} / \mathrm{Kg} \pm 0.01 \mathrm{SD}$ over the entire barrier (Supplementary Material 10). At $80 \mathrm{~L} / \mathrm{s}$, these values decreased to a mean velocity of $0.69 \mathrm{~m} / \mathrm{s} \pm 0.02 \mathrm{SD}$ and mean TKE of $0.01 \mathrm{~J} / \mathrm{Kg} \pm 0.00 \mathrm{SD}$. There was a large increase in mean TKE (860\%) between 105 and $130 \mathrm{~L} / \mathrm{s}$, whilst the mean velocity only increased by $29.47 \%$ between these discharges. In comparison, much smaller increases in velocity $(1.45 \%)$ and TKE (37.14\%) were observed between 80 and $105 \mathrm{~L} / \mathrm{s}$.

Step 2: forces experienced by preserved fish over the prototype barrier

We found gudgeon experienced significantly lower 3D-forces (mean $0.230 \mathrm{~N} \pm 0.116$ ) than the other species (round goby: $0.298 \mathrm{~N} \pm 0.134$, bullhead: $0.264 \mathrm{~N} \pm 0.084$ ) at $130 \mathrm{~L} / \mathrm{s}$ water discharge (Fig. 4 A), with no significant differences observed between round goby and bullhead (Supplementary Material 11). The corrected mean 3D-forces (reference force subtracted from the force measured for the fish) were $0.103 \mathrm{~N} \pm 0.134$ for round goby, $0.035 \mathrm{~N} \pm 0.116$ for gudgeon and $0.069 \mathrm{~N} \pm 0.084$ for bullhead at 130 $\mathrm{L} / \mathrm{s}$. At $80 \mathrm{~L} / \mathrm{s}$, we detected corrected mean 3D-forces of $0.049 \mathrm{~N} \pm 0.032$ for round goby, $0.067 \mathrm{~N} \pm 0.060$ for gudgeon and $0.060 \mathrm{~N} \pm 0.048$ for bullhead. When water discharge was increased from 80 to $130 \mathrm{~L} / \mathrm{s}$, the corrected mean 3D-forces for preserved round goby increased by $109 \%$ and $15 \%$ for bullhead, whilst they declined by $48 \%$ for gudgeon.

\section{Correlations between force and flow at $130 \mathrm{~L} / \mathrm{s}$ water discharge}

In general, small but significant correlation coefficients were observed between the forces experienced by the preserved fish and the flow velocities at the corresponding measurement points in the barrier (Fig. 4A). This suggests a weak linear relationship between force and flow velocity in the barrier: although the flow velocity varied by a range of $0.30 \mathrm{~m} / \mathrm{s}$ over the barrier, from a minimum of $0.81 \mathrm{~m} / \mathrm{s}$ (position a1) to a maximum of $1.11 \mathrm{~m} / \mathrm{s}$ (position $\mathrm{b} 1$ ), the forces measured for the preserved fish were similar between locations with strong and weak velocity. This suggests that the mean forces experienced by fish in the barrier did not correspond with the mean local flow velocity and the fish did not necessarily experience strong forces at locations with high velocity.

Step 3: swimming behaviour of live fish over the prototype barrier

In total, 930 fish trajectories over or near the barrier were recorded and analysed (Supplementary Material 12, 13). The 'passage' trajectories observed at $130 \mathrm{~L} / \mathrm{s}$ water discharge exhibited a homogenous spatial distribution over the barrier prototype for round goby and gudgeon (Fig. 5). In contrast, bullhead passed straight along the sides of the barrier at $130 \mathrm{~L} / \mathrm{s}$ water discharge. Contrary to the 'uncompleted passage' trajectories of bullhead and gudgeon, most of the 'uncompleted passage' trajectories for round goby started at the upper right corner of camera 2 and left the lower left corner of the screen. This pattern, together with some round goby 'passage' trajectories entering the screen at the middle of the barrier (at 80 $\mathrm{L} / \mathrm{s}$ ), suggests round goby swam over the side walls of the barrier (Fig. 5). This behaviour was commonly observed for round goby and may have been performed to reduce the distance needed to swim along the barrier for successful passage. All fish returned with relatively straight paths (Fig. 5, Supplementary Material 12) and immediately left the barrier swimming to the left or the right. 

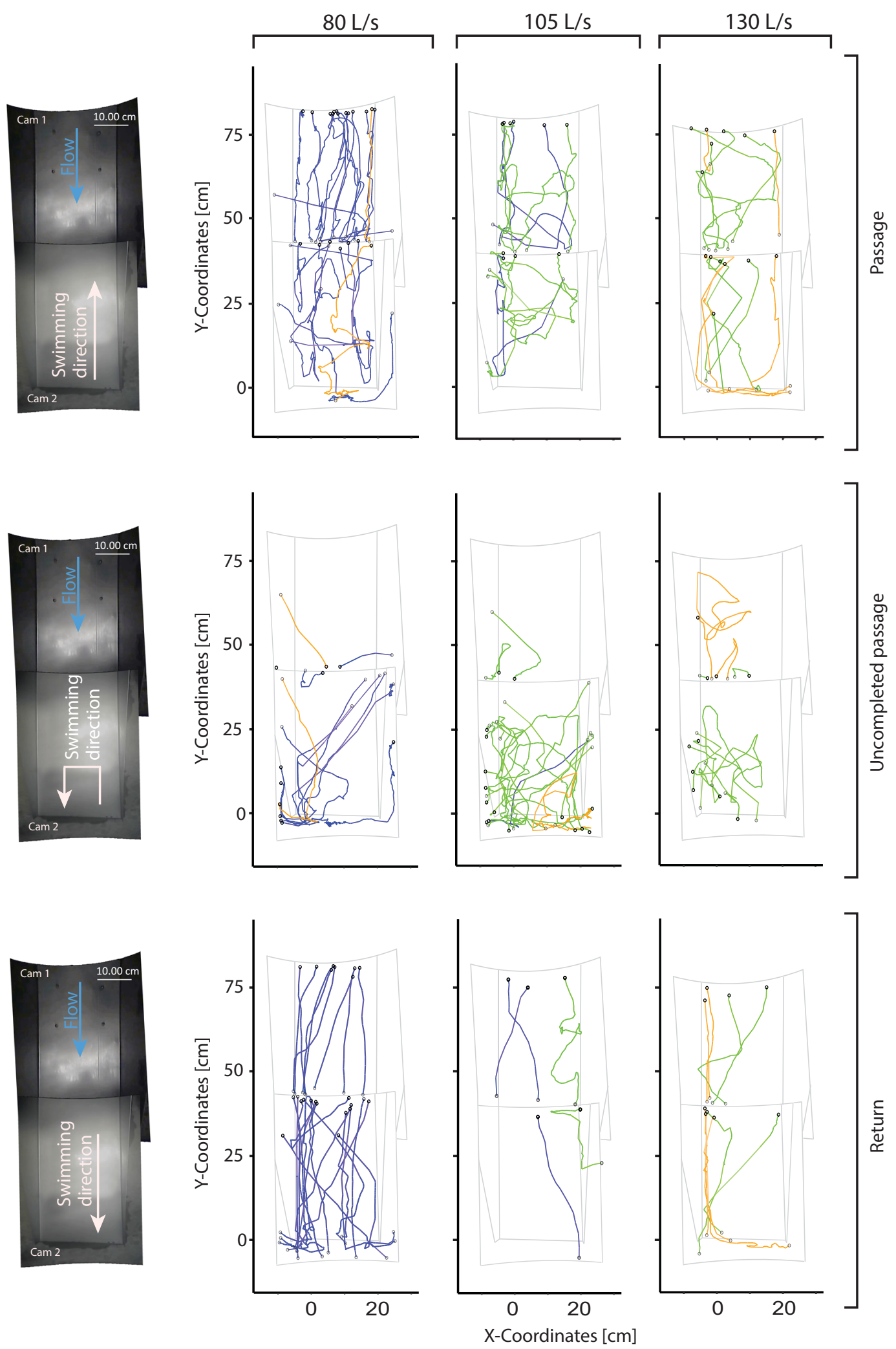
४Fig. 5 Maps of fish trajectories during passage, uncompleted passage and return events for round goby (violet), gudgeon (green) and bullhead (orange) over the barrier. The views of both cameras (Cam 1 and Cam 2) are provided on the left

\section{Principal component analysis}

The first principal component of the PCA explained $37.94 \%$ of the variation of all recorded trajectories and was best represented by the indices describing swimming speed ('mean speed', 'SD speed', 'maximum acceleration'), whilst the second principal component explained $18.06 \%$ of the variation and was best represented by the 'straightness' and 'sinuosity' indices. In general, the overlaid confidence ellipses in the PCA revealed the similarities of the trajectories assessment indices between the three species (Supplementary Material 14). The PCA was performed to obtain a general visual overview of the entire dataset based on $95 \%$ confidence ellipses. In general, the PCA suggested that the indices describing swimming speed ('mean speed', 'SD speed', 'maximal acceleration') better explain the variations in the 'passage' trajectories events for all species than 'sinuosity' and 'straightness'.

\section{Random forest machine learning approach}

The random forest model predicted the species based on the trajectory features, the event type and the water discharge with an overall mean accuracy of $64.68 \%$. For the different species, we achieved an accuracy of $85.24 \%$ for round goby, for gudgeon $92.16 \%$ and for bullhead $16.67 \%$ (Fig. 6). This shows that we were able to identify especially gudgeon and round goby only with information on swimming patterns (as represented by the trajectory features), the event type and the water discharge on a reliable level. The low prediction accuracy in bullhead is assumed to result from the decreased proportion of training trajectories for bullhead (13.74\%) of the entire data set compared to round goby (47.33\%) and gudgeon (38.93\%).

With regard to the variable importance (represented by the mean decrease in accuracy when excluding the variable whilst training [mda]), we found the water discharge (mean $605.57 \mathrm{mda}$ ), distance (mean 112.80 mda) and event type (mean $55.32 \mathrm{mda}$ ) to be the most important variables for the accuracy of the model. The least important variables were variation in speed (mean $3.28 \mathrm{mda}$ ), mean speed (mean $23.28 \mathrm{mda}$ ) and sinuosity (mean $30.36 \mathrm{mda}$ ). The mean importance of the remaining predictor variables was 35.90 mda for straightness and $32.67 \mathrm{mda}$ for maximum acceleration.

\section{Comparison of 'passage' trajectories \\ between species and water discharges}

The feature boxplots for only the 'passage' trajectories revealed similar overall behaviour between species, with adaptions in swimming behaviour to increased water discharge (Fig. 7). For example, both round goby and gudgeon increased their mean swimming speed at the highest water discharge: the 'mean swimming speed' for round goby was $1.00 \mathrm{~m} / \mathrm{s}$ at 80 $\mathrm{L} / \mathrm{s}$ and increased by $48 \%$ to $1.48 \mathrm{~m} / \mathrm{s}$ at $105 \mathrm{~L} / \mathrm{s}$; a $116 \%$ increase in 'mean swimming speed' was observed for gudgeon from $0.91 \mathrm{~m} / \mathrm{s}(105 \mathrm{~L} / \mathrm{s})$ to $1.97 \mathrm{~m} / \mathrm{s}$ (130 L/s; Fig. 7). Similar trends were observed for the 'SD swimming speed', with a $28 \%$ increase from 0.88 to $1.13 \mathrm{~m} / \mathrm{s}$ (between 80 and 105 $\mathrm{L} / \mathrm{s}$ ) for round goby and $125 \%$ increase from 0.77 to $1.77 \mathrm{~m} / \mathrm{s}$ (between 105 and $130 \mathrm{~L} / \mathrm{s}$ ) for gudgeon. In addition, round goby and gudgeon displayed straighter trajectories at higher water discharge. The straightness increased by $21 \%$ from $0.71(80 \mathrm{~L} / \mathrm{s})$ to $0.86(105 \mathrm{~L} / \mathrm{s})$ for round goby and by $35 \%$ from $0.66(105 \mathrm{~L} / \mathrm{s})$ to 0.89 $(130 \mathrm{~L} / \mathrm{s})$ for gudgeon. Although both species exhibited similar adaptations to swimming performance in response to increased flow, these behavioural changes appeared at different flow rates: between 80 and 105 $\mathrm{L} / \mathrm{s}$ for round goby and 105 and $130 \mathrm{~L} / \mathrm{s}$ for gudgeon. The absence of round goby 'passage' at the highest water discharge tested $(130 \mathrm{~L} / \mathrm{s})$ and absence of gudgeon 'passage' at the lowest water discharge tested $(80 \mathrm{~L} / \mathrm{s})$, together with their similar responses to increased flow, suggest both species use similar strategies to respond to the challenge of increased flow, but prefer different flow rates for passage. Round goby passed the barrier at weaker flow, whilst gudgeon passed at stronger flow. We excluded the bullhead from this comparison because only two trajectories crossing the camera screen (passage) were recorded at $80 \mathrm{~L} / \mathrm{s}$. 
Total data set

1. The data were separated after species

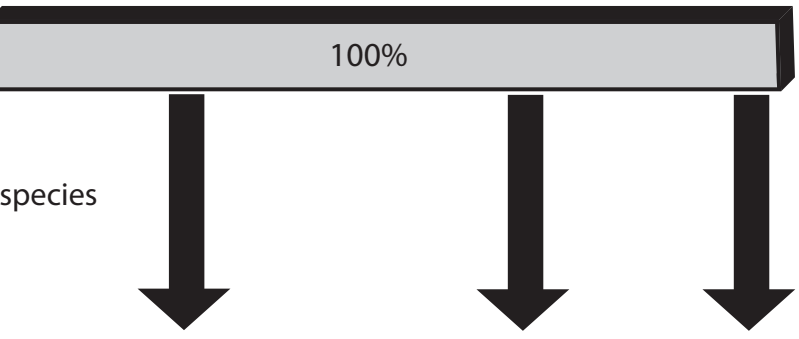

Round goby

Gudgeon

Bullhead

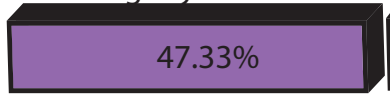

$38.93 \%$

$13.74 \%$

2. Three random data sets of similar size were created with equal species proportion

3. Always two data sets were combined to train models for prediction of the third data set
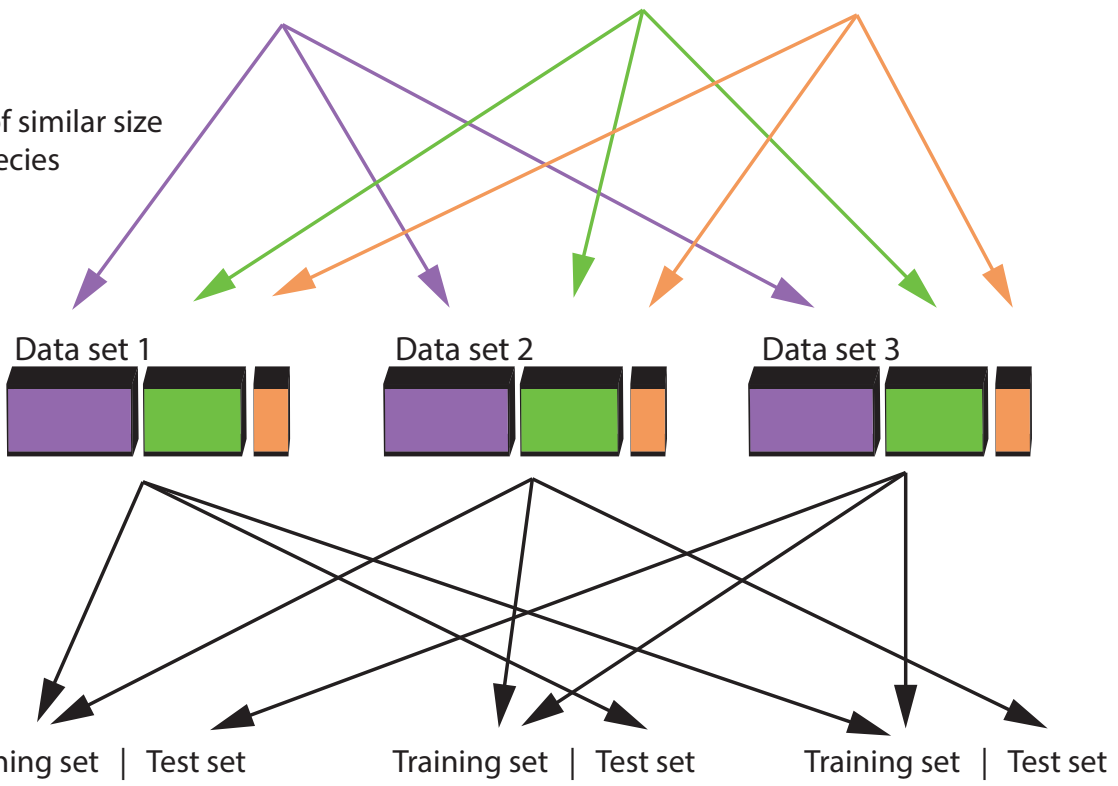

Training set | Test set

4. The models were trained and the
species of the test sets were
predicted
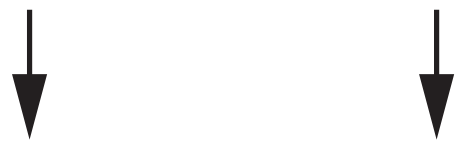

\begin{tabular}{|c|c|c|c|}
\hline \multirow{5}{*}{ 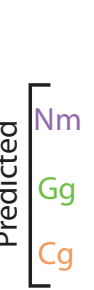 } & \multicolumn{3}{|c|}{ True } \\
\hline & $\mathrm{Nm}$ & $\mathrm{Gg}$ & $\mathrm{Cg}$ \\
\hline & 19 & 0 & 1 \\
\hline & 2 & 16 & 2 \\
\hline & 0 & 1 & 3 \\
\hline
\end{tabular}

Species accuracy [\%]:

$90.48 \quad 94.12 \quad 50.00$

Accuracy per model[\%]:

78.20

\begin{tabular}{c|c|c|c|}
\multicolumn{1}{c}{ Nm } & \multicolumn{1}{c}{ Gg } & \multicolumn{1}{c}{$\mathrm{Cg}$} \\
\cline { 2 - 4 } $\operatorname{Nm}$ & 20 & 0 & 1 \\
\cline { 2 - 4 } $\operatorname{Gg}$ & 1 & 16 & 5 \\
\cline { 2 - 4 } $\operatorname{cg}$ & 0 & 1 & 0 \\
\cline { 2 - 4 } & &
\end{tabular}

$95.24 \quad 94.12 \quad 0.00$

63.12

\begin{tabular}{c|c|c|c|}
\multicolumn{1}{c}{$\mathrm{Nm}$} & $\mathrm{Gg}$ & \multicolumn{1}{c}{$\mathrm{Cg}$} \\
\cline { 2 - 4 } $\operatorname{Nm}$ & 14 & 0 & 2 \\
\cline { 2 - 4 } $\operatorname{Gg}$ & 4 & 15 & 4 \\
\cline { 2 - 4 } $\operatorname{cg}$ & 2 & 2 & 0 \\
\cline { 2 - 4 } & &
\end{tabular}

$\begin{array}{lll}70.00 \quad 88.24 & 0.00\end{array}$

52.74

Mean accuracy [\%]: 
4Fig. 6 Cross validation was applied to assess the accuracy of the random forest models trained with all trajectories recorded for round goby $(\mathrm{Nm})$, gudgeon $(\mathrm{Gg})$ and bullhead $(\mathrm{Cg})$ over the barrier. The confusion matrices (4.) illustrate the number of correctly (grey boxes) and mispredicted (white boxes) trajectories

\section{Discussion}

The flow force behaviour approach: filling the research gap

In this study, we tested a prototype hydraulic barrier by (i) describing the flow field created, (ii) assessing the physical impact of the flow field on the bodies of preserved fish of three target species, and (iii) analysing the swimming behaviour of live fish over the barrier in the same flow field. Overall, we aimed to evaluate whether the hydrodynamic conditions within the barrier can selectively prevent the upstream migration of an invasive fish species. Our combined approach revealed: The hydrodynamic burdens experienced by the fish differed significantly between species. The fishes' swimming behaviour in the flow field over the barrier was distinct enough to identify the fish species alone with information about the trajectories and the water discharge on a reliable level. In addition, the live fish responded with faster swimming speeds and straighter trajectories to the increased hydraulic forces experienced at higher water discharges. Gudgeon passed the barrier most frequently at $130 \mathrm{~L} / \mathrm{s}$ water discharge. This behavioural observation can be well connected to a significantly smaller hydraulic burdens experienced by gudgeon at $130 \mathrm{~L} / \mathrm{s}$ water discharge compared to the other species.

These findings show that the species differed in their swimming behaviour when swimming across the barrier but had a similar behavioural response to the increased hydraulic burden at higher water discharge: Especially round goby and gudgeon swam faster and straighter across the barrier at increased water discharges.

Round goby did not pass the barrier at $130 \mathrm{~L} / \mathrm{s}$ water discharge, contrary to gudgeon and bullhead, whilst round goby experienced stronger forces than gudgeon over the barrier. This suggests that the prototype hydraulic barrier created species-selective hydraulic conditions in the vertical slot fish pass. These conditions might have prevented the passage of round goby but enabled passage of gudgeon and bullhead in our experiments.

Step 1: the flow field over the prototype selective barrier

Our flow measurements indicated homogenous flow over the centre of the barrier and higher velocities and TKE values at both ends of the barrier. The flow field within a vertical slot without the barrier is described in the literature as being similar to that of a turbulent jet plane with a rapid longitudinal decay (Liu et al., 2006). Our barrier prototype was designed to separate this jet and the corresponding shear layer from the flow field in the basin over an extended distance to create a selective barrier effect. The acoustic Doppler measurements indicated the prototype successfully extended the turbulent jet plane. However, similarly to Wiegleb et al. (2020), we were not able to measure the flow in the vicinity of the side walls or very close to the bottom of the barrier, as the smooth surface of the barrier reflected the acoustic signals and reduced the quality of the measurements close to the walls. Haro et al. (2004) described the flow field in a smooth surface rectangular flume with reduced flow and secondary vortex systems along the edges, which may also occur within our barrier. Indeed, the corners in our prototype barrier provide suitable flow conditions for bullhead, as this was the only species that exhibited a clear tendency to swim along the edges when swimming across the barrier.

Step 2: force measurements-a key ingredient of understanding the interactions between flow and fish

Force measurements represent a key link between the flow measurements and observations of live fish behaviour and provide important insight into the species-specific hydraulic burdens experienced by the fish during their passage. While the flow speed varied in the vicinity of the barrier surface compared to the forces, the forces experienced by the preserved fish remained similar between measurement positions. This suggests that the forces experienced by the fish are not solely determined by the mean flow velocity, although a strong relationship between flow velocity and experienced drag force was previously reported 
Fig. 7 Trajectory features of 'passage' events for the three fish species. The number of completed passage events recorded for each species is provided at the top of the figure. The boxplot centres represent the median and the values for the individual trajectories are represented by the individual points

under standardized conditions in a flow channel (Wiegleb et al., 2020). However, this relationship was based on force measurements performed at one position in the flow field (Wiegleb et al., 2020), whilst the force data in the present study were obtained from several measurement points with different hydraulic and geometric boundaries. That we accounted for several measurement points is one possible explanation for the weak correlation between the force and flow data: the present study reveals the flow field varies in the vicinity of the barrier surface, as reported in previous flow studies in open flume channels (Haro et al., 2004; Wiegleb et al., 2020). Another reason for the weak correlation may be the complex shape of the fish. For example, flow from the side encountered a larger surface of body and tail than in case of flow from the front, when only the head surface of the fish was exposed to the flow. Therefore, the 3D-forces strongly depend on the direction of the flow encountered by the fish. This is an important aspect of this study that differs from measurements of one-dimensional drag force alone (one axis sensor (Wiegleb et al., 2020)).

In general, we observed gudgeon experienced significantly smaller forces compared to round goby and bullhead at $130 \mathrm{~L} / \mathrm{s}$ water discharge. One reason may be that gudgeon have a more streamlined body shape. Variations in body shape can alter the drag forces experienced by fish: pregnant female guppies (Poecilia reticulata Peters, 1859) experience much higher drag forces than similarly sized non-pregnant females (Quicazan-Rubio et al., 2019). Furthermore, it is possible that the flow conditions support passive propulsion of gudgeon (Liao et al., 2003; Beal et al., 2006). However, it should be noted that the forces could not be measured directly on the ground, as placing the preserved fish in contact with the ground would have induced uncontrolled friction forces (Wiegleb et al., 2020).

The force measurements described the general physical impact of flow on the fish body over the barrier under standardized conditions. Research assumes that live fish have swimming modes
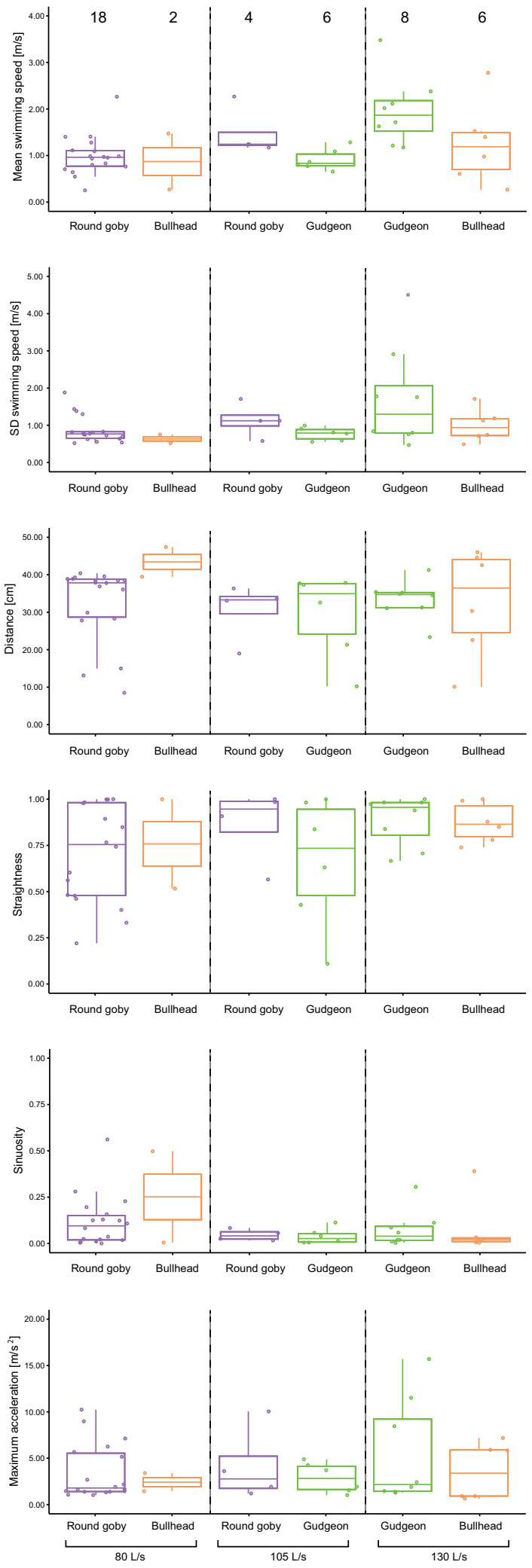
corresponding to their body shape and locomotor mode (Blake, 2004). The locomotor modes of the fish we applied here were similar, especially between round goby and bullhead (Egger et al., 2020). Of course, by testing preserved fish, we were not able to account for kinematic modulations induced by movements of the fish. Therefore, it is possible that the live fish actually experienced lower forces because they adapted their swimming behaviour to the local flow conditions. Another point is that all objects have eigenfrequency and these are more or less excited during different water velocities and resulting disturbances at our force measurements. Knowing that muscle tone of the fish will also lead to a change in eigenfrequency of the fish oscillations, preserved fish will nevertheless provide a valid indication how resistance changes with increasing water velocity, also based on induced oscillations and resulting water resistance. In addition, turbulence has been reported to potentially have strong impact on the fish swimming performance. On the one hand, because of destabilizing effects at specific relationships between vortex and fish size (Lupandin, 2005) and on the other hand, because of potential energetic support due to passive propulsion at specific vorticity (Beal et al., 2006). Due to the relatively small measurement volume of the acoustic Doppler point measurements $\left(7 \mathrm{~mm}^{3}\right)$ however, such vortices with increased effect on the fish swimming performance of our tested fish were not detectable by our flow measurements, whilst their effect on the fish body was detected by the force measurements. Therefore, we assume that the fish body acts as a transducer that displays flow characteristics with more relevance to fish swimming than the acoustic Doppler measurements. We propose force measurements with preserved fish or artificial models should be considered for future flow assessments to enable more precise characterization of the suitability of flow fields for specific species, than numerical modelling of forces alone.

Step 3: behaviour of live fish over the barrier: general adaptations in speed, speed variation and straightness at increased flow

Modern swimming performance tests are commonly based on enforced swimming and strict protocols of tested velocities (Tierney et al., 2011; Egger et al., 2020), whilst the observation of the free movement and voluntary ascending behaviour of the tested fish was an important quality of our experiments. Therefore, we were able to perform reliable predictions for the fish species only with information about the videorecorded voluntary fish trajectories in combination with the water discharge. Indeed, to increase the precision of the random forest model, we excluded the 'approach' trajectories from the random forest analysis. Together with the PCA, which included all trajectories and suggested rather low variation in the fish trajectories, we conclude that the species behaved similarly when approaching the barrier but that differences in the swimming behaviour were increased when the fish were observed over the barrier (whilst passage, return or uncompleted passage). These differences were strong enough that the trained random forest model was able to distinguish reliably between the trajectories of round goby and gudgeon. The low model accuracy for bullhead might result from the low proportion of bullhead trajectories from the entire training set. It might be possible to increase the bullhead prediction accuracy by increasing the number of bullhead trajectories in the model training set, but this requires the recording of more bullhead trajectories.

Having a closer look at the trajectories of fish that completely passed the barrier (passage trajectories), we observed on the one hand a clear difference in the preferred water discharge for passage between the species, especially between round goby (preferred 80 $\mathrm{L} / \mathrm{s}$ ) and gudgeon (preferred $130 \mathrm{~L} / \mathrm{s}$ ). This corresponds to the results of the random forest model, when 'water discharge' was the most important variable for discriminating between the species. On the other hand, we observed a very similar behaviour in all tested species: A general adaptation of the swimming trajectories was observed by increased speed, speed variation and straighter paths at increased flow. This observation corresponded to the random forest result, when 'speed variation', 'sinuosity and 'speed' had the smallest or medium importance for discrimination between species. Considering this similarity in behaviour between species, we conclude that all species behaved similar when challenging increased flow, but that the fish species differed in their preferred water discharge for passing the barrier and their swimming styles concerning the 'straightness' and the length of trajectories ('distance') over the barrier. 
Considering the variation in swimming behaviour between species, the significant differences between species in the forces experienced, and the observation that no round goby passed the barrier at the highest water discharge tested, we assume that the hydrodynamics created by the prototype barrier prevented the passage of round goby. Successful passage of gudgeon and bullhead at $130 \mathrm{~L} / \mathrm{s}$ water discharge supports the idea that such a barrier could provide a speciesspecific effect.

Random forest model: using the fish trajectories for species identification

To our knowledge, we were the first who published the identification of video-recorded fish in a vertical slot fish pass based on their swimming patterns using a modern machine learning approach. Indications for differences in swimming behaviour between species, especially in swimming speed and acceleration, have been reported previously by Rodríguez et al. (2015). That these differences can be used to identify different species reliably with information extracted from their swimming paths and the present water discharge was shown in our study.

This technique might improve fish species identification with poor image quality or increased water turbidity, because detailed records of fish body contours are not necessary for this approach, contrary to fish identification methods based on fish body shape recognition (Shafait et al., 2016). Indeed, we visually screened the videos and performed the tracking manually which was very time consuming. There are modern computer vision techniques available, which enable automated object detection and classification in videos (Han et al., 2018). A combination of computer vision techniques with automated fish identification based on swimming patterns would represent a promising tool for visual non-invasive fish pass monitoring. Because vertical slots in fish passes have to be passed by every fish passing the fish pass, vertical slots act as bottlenecks that can be monitored by camera systems, as described in our study and Belo et al. (2021). This might enable the reduction of more invasive techniques such as electro fishing (Knaepkens et al., 2005; Knaepkens et al., 2007) or implanted PIT-tags (Aarestrup et al., 2003), which implement a direct contact with the fish.
Evaluation of the random forest machine learning approach

With an accuracy of $92.16 \%$ for gudgeon and $85.48 \%$ for round goby, the model performed promisingly for these species. Indeed, the lower prediction accuracy in bullhead induced by the smaller amount of trajectories recorded for this species in our experiments represents one very important challenge when applying biological data to modern machine learning approaches. That our study aimed at observing exclusively voluntary swimming behaviour meant, that we did not record a data set perfectly suitable for training machine learning models, because of varying numbers of trajectories for the different species. Indeed, we showed that it is possible to identify fish based on swimming patterns extracted from underwater videos. With more data and further effort in the design and improvement of machine learning models, it might be possible to increase the identification accuracy in the future.

\section{Limitations of the video observations}

Two cameras were used to observe the entire prototype barrier. However, we were not able to connect the paths of the fish crossing both screens (e.g., 'passage') as we were not able to identify individual fish leaving the screen and entering the other-especially as, at the lowest water discharge tested, round goby swam over the side walls and into the barrier in the vicinity of the slot and were thereby only recorded by the upstream camera. This could be avoided by wide angle cameras in future experiments. Because of the possibility of recording the same fish multiple times, it is likely that the number of recorded events does not represent the actual number of recorded fish. Indeed, accepting this limitation actually creates an important strength of this study. As the fish were able to move freely through the fish pass model for an extended time of two hours and we avoided a "shooing effect" as well as a human presence during the experiments, we could observe voluntary swimming behaviour in a realistic fish pass setup. Furthermore, the three tested fish species are reported to predominantly swim near the ground at high velocities (Egger et al., 2020). However, vertical swimming was possible, but was not accounted for in our analysis due to the vertical views of both cameras that provided a planar projection of the fish paths over the ground. This issue could be avoided in future 
investigations by implementing additional cameras and performing tracking in 3D-space.

Evaluation of the prototype hydraulic barrier and relevant future research

The design of our prototype barrier to prevent the upstream passage of round goby follows the concept of a hydraulic barrier for round goby initially proposed by Hoover et al. (2003). The barrier evaluated in the present study has a simple construction and consists of a cut and shaped stainless-steel plate. Our experiments indicated that the prototype met the requirements (mentioned in the introduction) of sufficient length $(1.00 \mathrm{~m})$, flow velocity $(0.91 \mathrm{~m} / \mathrm{s})$ and smoothness (stainless steel, roughness $0.015 \mathrm{~mm}$ ) at a water discharge of $130 \mathrm{~L} / \mathrm{s}$; under these conditions, round goby were not able to pass the barrier whilst gudgeon and bullhead crossed the complete length of the barrier. This suggests the prototype barrier has a species-selective effect at a water discharge of 130 L/s. Indeed, Egger et al. (2020) reported a strong reduction of the passage rate for all species tested at the barrier compared to the previous untreated slot, suggesting a general passage-reducing effect of the barrier in all tested species.

Due to the diverse palette of methods applied in our approach, we recommend models of fish pass facilities be tested in the laboratory prior to implementation at dams and subsequent field assessments. We tested the prototype under laboratory conditions and assume that, in the field, vegetation growth or debris may alter the hydraulics and thereby impact the effectiveness of a barrier in a real fish pass over time. It should also be tested to what extent the video recordings are ready to be employed in the field. Further machine learning tests will show to what extent field conditions, such as turbidity and air bubbles, pose obstacles to identification of fish trajectories.

The prototype hydraulic barrier performed promisingly, though the mechanisms that such hydraulic barriers employ also have ecological ramifications. Studies have revealed that personality traits and motivation are relevant to the passage of round goby (Myles-Gonzalez et al., 2015; Hirschet al., 2017) and benthic fish swimming behaviour can vary in different seasons (Van Liefferinge et al., 2005). Therefore, evaluations of the actual impact of the barrier on benthic fish swimming behaviour require comparisons with an unaffected vertical slot and long-term field studies are necessary to test the performance of the barrier in a real fish pass. In addition, our data are representative for our scaled vertical slot fish pass model, but the barrier performance in fish passes of different type or dimensions will have to be assessed in further studies. However, this new evidence on benthic fish swimming and functionality, combined with the flow force behaviour approach applied in this study, are expected to inform the design and engineering of fish passes adapted to the requirements of specific ecosystems (Katopodis, 2005; Kemp, 2012).

Acknowledgements We gratefully thank Bernd Egger for fundamental help with the conception of this study and conducting the experiments. Furthermore, we thank Peter Reimann, Laurent Marot and Marco Martina, Department of Physics, University of Basel, for technical support; Hans-Peter Jermann, Kantonal Fisheries Officer, Basel Stadt, and Frank Hartmann and Stephan Hüsgen for help with catching the fish; and Michael Ritzmann, Christopher Ulrich and Adeline Pöschl, technical staff at the KIT, for their kind assistance. We especially thank Manuela Flattich and David Scherrer for numerous hours of video screening. We also thank Daniel Oesterwind, Thünen Institute of Baltic Sea Fisheries, and Gabriel Erni Cassola, Program MGU, University of Basel, for critical review of the manuscript, two anonymous reviewers for helpful advice, and Franziska Wiegleb for providing vital support.

Author contributions PBH conceived the study. GR provided the force measurement system and gave instructions in particular for the force measurements and the analysis of the random forest model. All authors conceptualized the research. JW and PEH performed the experiments. JW analysed the data, created the figures and wrote the first draft. All authors wrote and edited the manuscript. PBH supervised the study and acquired funding. All authors gave final approval for publication.

Funding This work was funded by the Federal Office for the Environment (FOEN; Contract-Nr. Q493-0660); Kantons Basel Stadt, as well as the Lotteriefunds of Basel Land, Aargau and Solothurn. The BIROMED-Lab, DBE, University of Basel, contributed funding for the force measurement system.

Availability of data Data are available in the Supplementary Material.

Code availability Code used to analyse the data is available in the Supplementary Material.

\section{Declarations}

Conflict of interest All authors declare that they have no conflict of interests. 
Consent to participate Not applicable.

Consent for publication Not applicable.

Ethical approval All animal experiments were approved by the Swiss cantonal authorities (permits Nr. 2934 and 2846) and the German regional authorities (permit Nr. G217_17-IWG).

Open Access This article is licensed under a Creative Commons Attribution 4.0 International License, which permits use, sharing, adaptation, distribution and reproduction in any medium or format, as long as you give appropriate credit to the original author(s) and the source, provide a link to the Creative Commons licence, and indicate if changes were made. The images or other third party material in this article are included in the article's Creative Commons licence, unless indicated otherwise in a credit line to the material. If material is not included in the article's Creative Commons licence and your intended use is not permitted by statutory regulation or exceeds the permitted use, you will need to obtain permission directly from the copyright holder. To view a copy of this licence, visit http://creativecommons.org/licenses/by/4.0/.

\section{References}

Aarestrup, K., M. C. Lucas \& J. A. Hansen, 2003. Efficiency of a nature-like bypass channel for sea trout (Salmo trutta) ascending a small Danish stream studied by PIT telemetry. Ecology of Freshwater Fish 12: 160-168.

Adrian-kalchhauser, I., A. Blomberg, T. Larsson, Z. Musilova, C. R. Peart, M. Pippel, ..., J. Y. Wilson, 2020. The round goby genome provides insights into mechanisms that may facilitate biological invasions. BMC Biology 18: 1-33.

Baki, A. B. M., D. Z. Zhu, A. Harwood, A. Lewis \& K. Healey, 2017. Rock-weir fishway I: flow regimes and hydraulic characteristics. Journal of Ecohydraulics 2: 122-141.

Beal, D. N., F. S. Hover, M. S. Triantafyllou, J. C. Liao \& G. V. Lauder, 2006. Passive propulsion in vortex wakes. Journal of Fluid Mechanics 549: 385-402.

Belletti, B., C. Garcia de Leaniz, J. Jones, S. Bizzi, L. Börger, G. Segura, ..., M. Zalewski, 2020. More than one million barriers fragment Europe's rivers. Nature 588: 436-441.

Belo, A. F., G. Cardoso, E. Pereira, B. R. Quintella, C. S. Mateus, C. M. Alexandre, ..., P. R. Almeida, 2021. Fish pass use by shads (Alosa alosa L. and Alosa fallax (Lacépède, 1803)): implications for monitoring and management. Ecohydrology. https://doi.org/10.1002/eco.2292.

Blake, R. W., 2004. Fish functional design and swimming performance. Journal of Fish Biology 65: 1193-1222.

Bombač, M., M. Četina \& G. Novak, 2017. Study on flow characteristics in vertical slot fishways regarding slot layout optimization. Ecological Engineering 107: 126-136.

Bombač, M., G. Novak, P. Rodič \& M. Četina, 2014. Numerical and physical model study of a vertical slot fishway. Journal of Hydrology and Hydromechanics 62: 150-159.

Carlson, R. L. \& G. V. Lauder, 2011. Escaping the flow: Boundary layer use by the darter Etheostoma tetrazonum
(Percidae) during benthic station holding. Journal of Experimental Biology 214: 1181-1193.

Community, B. O. (2017). Blender - a 3D modelling and rendering package. Amsterdam: Blender Foundation. https:// www.blender.org/

Drucker, E. G. \& G. V. Lauder, 1999. Locomotor forces on a swimming fish: three-dimensional vortex wake dynamics quantified using digital particle image velocimetry. Journal of Experimental Biology 202: 2393-2412.

Egger, B., J. Wiegleb, F. Seidel, P. Burkhardt-Holm \& P. Emanuel Hirsch, 2020. Comparative swimming performance and behaviour of three benthic fish species: the invasive round goby (Neogobius melanostomus), the native bullhead (Cottus gobio), and the native gudgeon (Gobio gobio). Ecology of Freshwater Fish 30: 391-405.

Fuentes-Pérez, J. F., A. T. Silva, J. A. Tuhtan, A. García-Vega, R. Carbonell-Baeza, M. Musall \& M. Kruusmaa, 2018. 3D modelling of non-uniform and turbulent flow in vertical slot fishways. Environmental Modelling and Software 99: 156-169.

Han, J., D. Zhang, G. Cheng, N. Liu \& D. Xu, 2018. Advanced deep-learning techniques for salient and category-specific object detection: a survey. IEEE Signal Processing Magazine 35: 84-100.

Haro, A., T. Castro-Santos, J. Noreika \& M. Odeh, 2004. Swimming performance of upstream migrant fishes in open-channel flow: a new approach to predicting passage through velocity barriers. Canadian Journal of Fisheries and Aquatic Sciences 61: 1590-1601.

Hirsch, P. E., M. Thorlacius, T. Brodin \& P. Burkhardt-Holm, 2017. An approach to incorporate individual personality in modeling fish dispersal across in-stream barriers. Ecology and Evolution 7: 720-732.

Hoover, J. J., Adams, S. R., \& Killgore, K. J. (2003). Can Hydraulic Barriers Stop the Spread of the Round Goby? Environmental Laboratory (U.S). https://hdl.handle.net/ $11681 / 5086$

Jansen, W., B. Kappus, J. Böhmer \& T. Beiter, 1999. Fish communities and migrations in the vicinity of fishways in a regulated river (Enz, Baden-Wurttemberg, Germany). Limnologica 29: 425-435.

Katopodis, C., 2005. Developing a toolkit for fish passage, ecological flow management and fish habitat works. Journal of Hydraulik Research 43: 451-467.

Katopodis, C. \& J. G. Williams, 2012. The development of fish passage research in a historical context. Ecological Engineering 48: 8-18.

Kemp, P. S., 2012. Bridging the gap between fish behaviour, performance and hydrodynmaics: an ecohydraulics approach to fish passage research. River Research and Applications 28: 403-406.

Kerr, J. R., A. S. Vowles, M. C. Crabb \& P. S. Kemp, 2021. Selective fish passage: restoring habitat connectivity without facilitating the spread of a non-native species. Journal of Environmental Management 279: 110908.

Knaepkens, G., K. Baekelandt \& M. Eens, 2005. Fish pass effectiveness for bullhead (Cottus gobio), perch (Perca fluviatilis) and roach (Rutilus rutilus) in a regulated lowland river. Ecology of Freshwater Fish 15: 20-29.

Knaepkens, G., E. Maerten \& M. Eens, 2007. Performance of a pool-and-weir fish pass for small bottom-dwelling 
freshwater fish species in a regulated lowland river. Animal Biology 57: 423-432.

Kornis, M. S., N. Mercado-Silva \& M. J. vander Zanden, 2012. Twenty years of invasion: a review of round goby Neogobius melanostomus biology, spread and ecological implications. Journal of Fish Biology 80: 235-285.

Larinier, M., 2008. Topologies and measurements of turbulent flow in vertical slot fishways. Hydrobiologia 609: 177-188.

Lauder, G. V. \& Æ. P. G. A. Madden, 2007. Fish locomotion: kinematics and hydrodynamics of flexible foil-like fins. Experiments in Fluids 43: 641-653.

Li, G., S. Sun, H. Liu \& T. Zheng, 2021. Schizothorax prenanti swimming behavior in response to different flow patterns in vertical slot fishways with different slot positions. Science of the Total Environment 754: 142142.

Liao, J. C., D. N. Beal, G. V. Lauder \& M. S. Triantafyllou, 2003. Fish exploiting vortices decrease muscle activity. Science 302: 1566-1569.

Liu, M., N. Rajaratnam \& D. Z. Zhu, 2006. Mean flow and turbulence structure in vertical slot fishways. Journal of Hydraulic Engineering 132: 765-777.

Lupandin, A. I., 2005. Effect of flow turbulence on swimming speed of fish. Biology Bulletin 32: 461-466.

McLean, D. J. \& M. A. Skowron Volponi, 2018. trajr: an R package for characterisation of animal trajectories. Ethology 124: 440-448.

Myles-Gonzalez, E., G. Burness, S. Yavno, A. Rooke \& M. G. Fox, 2015. To boldly go where no goby has gone before: boldness, dispersal tendency, and metabolism at the invasion front. Behavioral Ecology 26: 1083-1090.

Phillips, E. C., M. E. Washek, A. W. Hertel \& B. M. Niebel, 2003. The round goby (Neogobius melanostomus) in Pennsylvania tributary streams of lake erie. Journal of Great Lakes Research 29: 34-40.

Plesiński, K., A. Bylak, A. Radecki-Pawlik, T. Mikołajczyk \& K. Kukuła, 2018. Possibilities of fish passage through the block ramp: Model-based estimation of permeability. Science of the Total Environment 631-632: 1201-1211.

Porreca, A. P., W. D. Hintz \& J. E. Garvey, 2017. Do alluvial sand dunes create energetic refugia for benthic fishes? An experimental test with the endangered pallid sturgeon. River Research and Applications 33: 690-696.

Puertas, J., L. Pena \& T. Teijeiro, 2004. Experimental approach to the hydraulics of vertical slot fishways. Journal of Hydraulic Engineering 130: 10-23.

Quicazan-Rubio, E. M., J. L. Van Leeuwen, K. Van Manen, M. Fleuren, B. J. A. Pollux \& E. J. Stamhuis, 2019. Coasting in live-bearing fish: the drag penalty of being pregnant. Journal of the Royal Society Interface 16: 20180714.

Rahel, F. J. \& R. L. McLaughlin, 2018. Selective fragmentation and the management of fish movement across anthropogenic barriers. Ecological Applications 28: 2066-2081.

Ramler, D. \& H. Keckeis, 2020. Effects of hydraulic engineering restoration measures on invasive gobies in a large river (Danube, Austria). Biological Invasions 22: 437-453.

Rodríguez, Á., M. Bermúdez, J. R. Rabuñal \& J. Puertas, 2015. Fish tracking in vertical slot fishways using computer vision techniques. Journal of Hydroinformatics 17: 275-292.

Sagnes, P. \& B. Statzner, 2009. Hydrodynamic abilities of riverine fish: a functional link between morphology and velocity use. Aquatic Living Resources 22: 79-91.

Sanchez, P. X., X. Leng, J. Von Brandis-Martini \& H. Chanson, 2020. Hybrid modelling of low velocity zones in an asymmetrical channel with sidewall longitudinal rib to assist fish passage. River Research and Applications 36: 807-818.

Shafait, F., A. Mian, M. Shortis, B. Ghanem, P. F. Culverhouse, D. Edgington, ..., E. S. Harvey, 2016. Fish identification from videos captured in uncontrolled underwater environments. ICES Journal of Marine Science: Journal Du Conseil 73: 2737-2746.

Silva, A. T., M. C. Lucas, T. Castro-Santos, C. Katopodis, L. J. Baumgartner, J. D. Thiem, ...,S. J. Cooke, 2018. The future of fish passage science, engineering, and practice. Fish and Fisheries 19: 340-362.

Šlapanský, L., M. Janáč, K. Roche, P. Jurajda, M. Janáč \& K. Roche, 2020. Round goby movement patterns in a nonnavigable river. Canadian Journal of Fisheries and Aquatic Sciences 77(475): 483.

Thandiackal, R., C. H. White, H. Bart-smith \& G. V. Lauder, 2021. Tuna robotics: hydrodynamics of rapid linear accelerations. Journal of the Royal Society Proceedings B 288: 20202726.

Tierney, K. B., A. V. Kasurak, B. S. Zielinski \& D. M. Higgs, 2011. Swimming performance and invasion potential of the round goby. Environmental Biology of Fishes 92: 491-502.

Tsikata, J. M., M. F. Tachie \& C. Katopodis, 2014. Openchannel turbulent flow through bar racks. Journal of Hydraulic Research 52: 630-643.

United Nations. Convention on Biological Diversity, United Nations § (1992). http://www.cbd.int/doc/legal/cbd-en.pdf

Van Liefferinge, C., P. Seeuws, P. Meire \& R. F. Verheyen, 2005. Microhabitat use and preferences of the endangered Cottus gobio in the River Voer, Belgium. Journal of Fish Biology 67: 897-909.

Van Wassenbergh, S., K. Van Manen, T. A. Marcroft, M. E. Alfaro \& E. J. Stamhuis, 2015. Boxfish swimming paradox resolved: Forces by the flow of water around the body promote manoeuvrability. Journal of the Royal Society Interface 12: 20141146.

Wang, R. W., L. David \& M. Larinier, 2010. Contribution of experimental fluid mechanics to the design of vertical slot fish passes. Knowledge and Management of Aquatic Ecosystems 396: 02.

Wiegleb, J., P. E. Hirsch, B. Egger, F. Seidel \& P. BurkhardtHolm, 2020. Flow field-induced drag forces and swimming behavior of three benthic fish species. Limnologica 84 : 125812 .

Publisher's Note Springer Nature remains neutral with regard to jurisdictional claims in published maps and institutional affiliations. 